\title{
Trait Energy and Fatigue May Be Connected to Gut Bacteria among Young Physically Active Adults: An Exploratory Study
}

\author{
Ali Boolani ${ }^{1,2, *}$, Karyn M. Gallivan ${ }^{3}$, Kristin S. Ondrak ${ }^{3}$, Courtney J. Christopher ${ }^{4}$, Hector F. Castro ${ }^{4,5}$, \\ Shawn R. Campagna ${ }^{4,5}$, Christopher M. Taylor ${ }^{6}{ }^{(}$, Meng Luo ${ }^{6}$, Scot E. Dowd ${ }^{7}$, Matthew Lee Smith ${ }^{8,9}{ }^{\circledR}$ \\ and Lauri O. Byerley ${ }^{3,10, *}$
}

check for updates

Citation: Boolani, A.; Gallivan, K.M.; Ondrak, K.S.; Christopher, C.J.; Castro, H.F.; Campagna, S.R.; Taylor, C.M.; Luo, M.; Dowd, S.E.; Smith, M.L.; et al. Trait Energy and Fatigue May Be Connected to Gut Bacteria among Young Physically Active Adults: An Exploratory Study. Nutrients 2022, 14, 466. https:// doi.org/10.3390/nu14030466

Academic Editors: Ruggiero Francavilla and Franck Gael Carbonero

Received: 10 December 2021 Accepted: 18 January 2022 Published: 21 January 2022 Publisher's Note: MDPI stays neutral with regard to jurisdictional claims in published maps and institutional affiliations.

Copyright: (C) 2022 by the authors. Licensee MDPI, Basel, Switzerland. This article is an open access article distributed under the terms and conditions of the Creative Commons Attribution (CC BY) license (https:// creativecommons.org/licenses/by/ $4.0 /)$.
1 Department of Physical Therapy, Clarkson University, Potsdam, NY 13699, USA

2 Department of Biology, Clarkson University, Potsdam, NY 13699, USA

3 Sports and Health Sciences, School of Health Sciences, American Public University System, Charles Town, WV 25414, USA; kgallivan@apus.edu (K.M.G.); kristin.ondrak@mycampus.apus.edu (K.S.O.)

4 Department of Chemistry, University of Tennessee, Knoxville, TN 37996, USA; cleathe3@vols.utk.edu (C.J.C.); hcastrog@utk.edu (H.F.C.); campagna@utk.edu (S.R.C.)

5 Biological and Small Molecule Mass Spectrometry Core, University of Tennessee, Knoxville, TN 37996, USA

6 Department of Microbiology, Immunology and Parasitology, Louisiana State University Health Sciences Center, New Orleans, LA 70112, USA; ctay15@1suhsc.edu (C.M.T.); mluo2@lsuhsc.edu (M.L.)

7 Molecular Research LP, 503 Clovis Rd, Shallowater, TX 79363, USA; sdowd@mrdnalab.com

8 Department of Environmental and Occupational Health, School of Public Health, Texas A\&M University, College Station, TX 37916, USA; matthew.smith@tamu.edu

9 Center for Population Health and Aging, Texas A\&M University, College Station, TX 77807, USA

10 Department of Physiology, Louisiana State University Health Sciences Center, New Orleans, LA 70112, USA

* Correspondence: aboolani@clarkson.edu (A.B.); lauri.byerley1@mycampus.apus.edu (L.O.B.); Tel.: +504-319-5828 (A.B.); +704-340-4482 (L.O.B.)

Abstract: Recent scientific evidence suggests that traits energy and fatigue are two unique unipolar moods with distinct mental and physical components. This exploratory study investigated the correlation between mental energy (ME), mental fatigue (MF), physical energy (PE), physical fatigue (PF), and the gut microbiome. The four moods were assessed by survey, and the gut microbiome and metabolome were determined from $16 \mathrm{~S}$ rRNA analysis and untargeted metabolomics analysis, respectively. Twenty subjects who were $31 \pm 5 \mathrm{y}$, physically active, and not obese $\left(26.4 \pm 4.4 \mathrm{~kg} / \mathrm{m}^{2}\right)$ participated. Bacteroidetes (45\%), the most prominent phyla, was only negatively correlated with PF. The second most predominant and butyrate-producing phyla, Firmicutes (43\%), had members that correlated with each trait. However, the bacteria Anaerostipes was positively correlated with ME $(0.048$, $p=0.032)$ and negatively with $\mathrm{MF}(-0.532, p=0.016)$ and PF $(-0.448, p=0.048)$, respectively. Diet influences the gut microbiota composition, and only one food group, processed meat, was correlated with the four moods-positively with $\operatorname{MF}(0.538, p=0.014)$ and $\operatorname{PF}(0.513, p=0.021)$ and negatively with ME $(-0.790, p<0.001)$ and PE $(-0.478, p=0.021)$. Only the Firmicutes genus Holdemania was correlated with processed meat $(\mathrm{r}=0.488, p=0.029)$. Distinct metabolic profiles were observed, yet these profiles were not significantly correlated with the traits. Study findings suggest that energy and fatigue are unique traits that could be defined by distinct bacterial communities not driven by diet. Larger studies are needed to confirm these exploratory findings.

Keywords: trait mental fatigue and energy; trait physical fatigue and energy; gut microbiome; gut microbiota

\section{Introduction}

Approximately $45 \%$ of the United States (US) population experiences elevated and persistent fatigue, a common, costly, and poorly understood problem [1-3]. It has been estimated that fatigue costs employers over $\$ 136$ billion per year in lost productivity [4]; however, these estimates do not account for fatigue-related driving and other accidents $[5,6]$, 
poor medical performance [7], school absences [8,9], and declines in school performance and negative health outcomes [9]. Fatigue is underreported in medical care [10] and linked to many diseases and disorders [3]. Despite fatigue's high financial and social costs, it is a poorly understood problem despite there being over 250 different instruments and no consensus about how best to measure fatigue [11]. One challenge for fatigue researchers is articulating the conceptual relationship between fatigue and energy.

Until recently, both colloquially and in research, feelings of fatigue were usually defined as "a lack of energy," suggesting that energy and fatigue were opposite ends of the same continuum. However, recent evidence suggests that energy and fatigue are two unique unipolar moods with distinct neurohormonal [12] and physiological [13,14] correlations. Further complicating this matter is evidence that suggests there are distinct mental and physical components of energy and fatigue (e.g., there are four moods, mental energy (ME), mental fatigue (MF), physical energy (PE), and physical fatigue (PF)) [15-17], and recent literature makes an additional distinction between the concepts of state and trait mental and physical energy and fatigue. As is the case with all moods, the state aspect of energy and fatigue are considered transient (short-term) and can be influenced by outside factors such as physical activity [18,19], sleep [20], and certain foods [21,22]. Recent evidence suggests that there is a trait aspect of energy and fatigue $[15,16,23]$, which modifies the effects of various interventions on state energy and fatigue $[15,23]$, objective measures of energy and fatigue [23], fine motor control [23], and other moods such as anxiety and depression [23].

In 2018, Loy and colleagues [12] provided evidence that state energy was associated with dopamine, while state fatigue was associated with serotonin, histamine, and inflammatory cytokines. Subsequently, Boolani and colleagues [13] provided cross-sectional evidence that peripheral mitochondrial function and normalized resting metabolic rate (nRMR) were associated with feelings of state energy; however, they did not report any physiological associations with feelings of state fatigue. Dupree and colleagues [14], in a case-controlled interventional study, reported changes in salivary Annexin A1 with feelings of ME, but no changes in salivary biomarkers were reported with feelings of MF. In 2017, Eshragh and colleagues [24] reported unique and overlapping epigenetic associations between feelings of energy and fatigue measured over two-time points. While this study [24] measured state energy and fatigue, as participants were asked how they felt in the moment, the stability of the measures over 6 months suggests that the study actually captured the trait aspect of energy and fatigue. However, these researchers [24] did not explicitly use a trait measure of energy and fatigue and, additionally, they did not differentiate between the mental and physical aspects of energy and fatigue. All of these studies suggest there may be unique yet overlapping biologic correlates of trait energy and fatigue that require further examination.

The human gastrointestinal tract contains thousands of bacterial species, primarily anaerobes, from two predominant phyla, Firmicutes and Bacteroidetes [25,26]. Most are located in the large bowel, where they ferment nondigestible food, making available nutrients and other substrates, like the short-chain fatty acid butyrate $[27,28]$. These processes, among others, performed by the gut microbiota are essential for maintaining homeostasis and normal gut physiology [29]. Several diseases have been linked to gut microbiota dysbiosis, such as obesity, coronary heart disease, diabetes, and inflammatory bowel disease [30]. The gut microbiota has also been implicated in mental health and cognition, and the existence of the gut-brain axis is well established [31].

Diet is one of the predominant influencers of gut microbiota composition. It determines the relative abundance of many microbial species, and these changes in microbial composition influence metabolic processes, and subsequently, the metabolome [32,33]. For example, long-term dietary patterns, particularly the intake of protein and animal fat (Bacteroides) versus carbohydrates or plant-based foods (Prevotella), are associated with so-called enterotypes [34]. Plants are rich in fiber and anti-inflammatory compounds, like polyphenols, which enter the colon where colonic microbiota convert them to bioavailable and biologically active compounds (e.g., apples) [35]. Evidence suggests that consuming 
polyphenol-rich diets may be associated with increased feelings of mental energy [16,36]. In addition, Mediterranean diets have been known to reduce fatigue; however, the authors did not differentiate between mental and physical fatigue [37]. Taken together, it may be hypothesized that diets associated with increased feelings of energy or reduced feelings of fatigue may also be associated with gut microbiome changes.

Recently, several studies reported an association between gut microbiome and feelings of fatigue [38-41]; however, these studies did not distinguish between the mental and physical aspects of fatigue. These preliminary findings have reported reduced diversity and altered gut microbiome among patients with cancer-related fatigue $[38,40]$ and myalgic encephalomyelitis/chronic fatigue syndrome (ME/CFS) [39,41]. These studies were exploratory in nature, with some studies not adjusting analyses to account for multiple comparisons $[38,40,41]$ and others comparing ME/CFS patients to healthy control populations [39,41]. One limitation of these recent studies is that findings have cannot be extrapolated to healthy populations. Another limitation is that previous studies [38-41] have measured fatigue as a lack of energy rather than measuring energy and fatigue as two separate unipolar moods with their own unique mental and physical components. Taken together, these limitations suggest there is a need to examine the association between gut microbiome diversity and feelings of mental and physical energy and fatigue uniquely among healthy individuals. Due to the limitations of current literature and the costs of performing these analyses, it is advisable to perform an exploratory study that may provide researchers with interesting findings without worrying about false positives to guide future targeted studies [42]. Therefore, this exploratory study will uniquely add to the literature by documenting the gut microbiota that may be correlated with the four distinct trait aspects of mental energy (ME), mental fatigue (MF), physical energy (PE), and physical fatigue (PF).

\section{Materials and Methods}

\subsection{Subjects}

Subjects were recruited from thirty-nine individuals who previously participated in a study investigating the gut microbiome. The potential subjects were contacted by email and invited to complete a brief survey about traits mental and physical energy and fatigue. The email described the study and explained that their survey responses would be correlated with their fecal microbiome. Individuals were excluded if they met any of the following criteria: (1) took an antibiotic over the last three months; (2) consumed an anti-diarrhea medicine in the last week; (3) took a laxative in the last week; (4) consumed prebiotics in the last week; (5) consumed probiotics in the last week; (6) been diagnosed with cancer; (7) been diagnosed with Crohn's disease; (8) taking prescription medications other than oral contraceptives; (9) cutting weight for an upcoming competition; (10) under the age of 25 years; or (11) lived outside the contiguous United States. Informed consent was obtained from all subjects involved in the study. A total of 20 subjects consented and completed the survey. All study procedures were approved by the Institutional Review Board at the American Public University System (2021-045-OL, 14 April 2021).

\subsection{Survey}

The survey was conducted online using Qualtrics (Qualtrics, XM, Provo, UT, USA). The mental and physical state and trait energy and fatigue scales [18] were used to discriminate between mental and physical energy and fatigue. The reliability and temporal stability of these scales has been previously demonstrated [21-23,43-47]. For the current study, reliability was tested using Cronbach's Alpha test in SPSS (IBM Corp. Released 2020. IBM SPSS Statistics for Windows, Version 27.0. Armonk, NY, USA: IBM Corp). The scores were PE, 0.767, PF, 0.899, ME, 0.890, and MF, 0.893. 


\subsection{Diet Recall}

The subjects were asked to recall the foods they ate the $24 \mathrm{~h}$ prior to collecting their fecal sample using an automated, web-based, self-administered $24 \mathrm{~h}$ dietary assessment (ASA24) (https:/ / epi.grants.cancer.gov/asa24/) (accessed on 1 January 2022) [48]. The program is freely available and can be accessed on the internet and mobile devices.

Fecal sample collection and DNA isolation: Each subject participated in an earlier study in which a stool sample was collected, the DNA was isolated, and the bacteria DNA was analyzed to identify the microbes present. Briefly, for that study, the DNA was extracted using the QIAamp DNA Stool Mini Kits (Qiagen, Germantown, MD, USA) modified to include bead-beating and RNase A treatment. A negative control was set for checking any potential bacterial DNA existing in chemicals or involved during the DNA extraction process. Purity and quantity were determined using a Thermo Scientific ${ }^{\mathrm{TM}}$ NanoDrop ${ }^{\mathrm{TM}}$ spectrophotometer (ThermoFisher Scientific, Waltham, MA, USA).

\subsection{Microbial Community Analysis}

Two amplification steps were performed to prepare a sequencing library using the AccuPrime Taq high-fidelity DNA polymerase system (Invitrogen, Carlsbad, CA, USA). A negative control with the control from DNA extraction and a positive control of Microbial Mock Community HM-276D (BEI Resources, Manassas, VA, USA) were set during amplicon library preparation. Next, $16 \mathrm{~S}$ ribosomal DNA hypervariable region V4 was amplified using genomic DNA and the gene-specific primers with Illumina adaptors: Forward 5'TCGTCGGCAGCGTCAGATGTGTATAAGAGACAG GTGCCAGCMGCCGCGGTAA3'; Reverse 5' GTCTCGTGGGCTCGGAGATGTGTATAAGAGACAG GGACTACHVGGGTWTCTAAT 3’. Polymerase chain reaction (PCR) products were purified using AMPure XP beads, and the purified amplicon DNA was amplified using the primers with different molecular barcodes: forward 5' AATGATACGGCGACCACCGAGATCTACAC [i5] TCGTCGGCAGCGTC 3'; reverse 5' CAAGCAGAAGACGGCATACGAGAT [i7] GTCTCGTGGGCTCGG 3'. The indexed amplicon libraries purified using AMPure XP beads and quantified using Quant-iT PicoGreen (Invitrogen) were normalized and pooled. The pooled library was quantified using KAPA Library Quantification Kit (Kapa Biosystems), diluted, and denatured according to Illumina's sequencing library preparation guidelines. In addition, 10\% PhiX was added to the sequencing library as an internal control and increased 16S RNA amplicon library diversity. The paired-end sequencing was performed on an Illumina MiSeq (Illumina, San Diego, CA, USA) using the $2 \times 250$ bp V2 sequencing kit.

Raw fastq files were processed using QIIME2 with the DADA2 plugin [49]. Forward and reverse reads were truncated to a uniform length of $240 \mathrm{bp}$, and $20 \mathrm{bp}$ were trimmed off of the front of each read to remove the primer. Amplicon sequence variants (ASVs) identified by DADA2 were merged, and any that fell out of the expected 250-255 bp length were discarded. Contingency-based filtering was performed to remove any ASVs that appeared in only one sample, and the consensus method was used to remove chimeric ASVs. A phylogenetic tree for diversity analysis was built by aligning remaining ASVs using mafft [50] and fasttree [51]. Taxonomic classification was performed using Greengenes v13.8 [52]. After primary data analysis, the remaining reads were analyzed using QIIME2 (Quantitative Insights Into Microbial Ecology) [53].

Read counts ranged from 14,628 to 90,465 , with an average read count per sample of 56,041. Alpha rarefaction was performed at a level of 14,600 reads.

Prediction of Metabolic Profile: Potential microbial functions were identified from the $16 S$ sequencing data. The raw data were formatted and imported into QIIME2. The dereplicated feature table and representative sequences were then used for closed-reference clustering against the Greengenes 13_5 97\% OTUs reference database. The closed-reference OTU table was used as input into the PICRUSt [54] pipeline, and the resulting PICRUSt metagenome data were further analyzed using STAMP (Statistical Analysis of Metagenomic Profiles) [55] using pathways labeled at Level 2. 


\subsection{Metabolomics}

Samples from the 20 survey subjects were processed at the Biological and Small Molecule Mass Spectrometry Core (BSMMSC), University of Tennessee, Knoxville, TN, USA (RRID: SCR_021368). Samples were pre-weighed ( $50 \mathrm{mg}$ aliquots) and extracted in biological triplicate. Briefly, water-soluble metabolites were extracted from fecal samples using an acidic acetonitrile extraction procedure [56]. An untargeted metabolomics method was employed to analyze the fecal microbiome using ultra high-performance liquid chromatography coupled to high-resolution mass spectrometry (UHPLC-HRMS). A 25 min method using a water:methanol solvent system with tributylamine as an ion-pairing reagent was used for reverse-phase chromatographic separation. This was accomplished by using a Synergi $2.6 \mu \mathrm{m}$ Hydro RP column $(100 \mathrm{~mm} \times 2.1 \mathrm{~mm}, 100 \AA$; Phenomenex, Torrance, CA, USA) and an UltiMate 3000 pump (Dionex). Eluted analytes were then ionized via negative mode electrospray ionization, and mass spectral analysis was accomplished using a Thermo Scientific Exactive Plus Orbitrap (San Jose, CA, USA) operating in full-scan mode [57,58]. Raw spectral files were converted to mzML files using the msCovert package from ProteoWizard [59]. Metabolites were identified manually by exact mass $( \pm 5 \mathrm{ppm})$ and retention time using an in-house library of metabolites and the open-source software, metabolomics analysis and visualization engine (MAVEN) [60,61]. There were 170 identified metabolites from the untargeted metabolomics analysis.

\subsection{Statistical Analysis}

Microsoft Excel (Office 365) and SPSS (IBM Corp. Released 2020. IBM SPSS Statistics for Windows, Version 27.0. Armonk, NY, USA: IBM Corp) were used for data analyses. Variables were evaluated for normality of distribution using a combination of histograms and the Shapiro-Wilks tests for normality. Neither the trait variables nor the gut microbiome data were normally distributed. Trait fatigue (both mental and physical) was positively skewed, while trait mental energy (both mental and physical) was negatively skewed. This skewness is similar to what has been reported in previous studies using these constructs $[15,23]$. Exponential, power, arscine, and logarithmic transformation techniques were attempted for all skewed variables; however, these transformations did not result in normally distributed data $(p>0.05)$, and the histograms did not differ much from the original. Thus, non-parametric analyses were used. Data were expressed as medians and interquartile range, and the actual $p$-values were reported. Relationships with $p<0.05$ were considered statistically significant. Spearman's rho coefficients were calculated to determine correlations between each trait (MF, ME, PF, and PE), bacteria species, diversity, predicted functional pathways, dietary nutrients, and food groups. Additionally, Spearman's rho was used to identify the correlation between the bacterial species and the traits, as well as the correlation between the nutrients and food groups correlated with the traits. Cronbach's Alpha coefficients were computed to test reliability of the trait measures.

Metabolomics spectral data were normalized by weight for each sample. The peak intensities were normalized by the weight of the fecal matter used in each aliquot $(\sim 50-120 \mathrm{mg})[62,63]$. The normalized data were used for principal component analysis (PCA), an unsupervised dimensionality reduction tool. MetaboAnalyst 5.0 was used to generate PCA plots, where the normalized data were filtered by interquartile range, log-transformed, and Pareto scaled [64]. The PCA plot partitioned the subjects into two clusters. To determine if specific traits (MF, ME, PF, PE) corresponded with each metabolic cluster, the subjects were assigned a group based on their clustering location. Normality of distribution for each trait was checked using a combination of histograms and Shapiro-Wilks tests. Data were abnormally distributed and exponential, power, arscine, and logarithmic transformation techniques were used to transform the data. None of the techniques produced normally distributed data in either group. Therefore, to determine differences in trait scores between the two clusters, Mann Whitney-U tests were used to compare the trait survey responses between each cluster. 
This was an exploratory study to identify unique relationships and guide future research efforts, so unadjusted findings are reported. If the Benjamini-Hochberg False Discovery Rate (FDR) of 30\% was used to correct for multiple tests post hoc, then none of the values in this study would be statistically significant.

\section{Results}

\subsection{Subject Description}

The average age of the 20 subjects ( 14 males, 6 females) was $31.1 \pm 5.0$ years. Their height, weight, and BMI were $67.7 \pm 4.4$ inches, $172.1 \pm 33.5 \mathrm{lbs}$, and $26.4 \pm 4.4 \mathrm{~kg} / \mathrm{m}^{2}$, respectively. Although the mean BMI was in the overweight category, the subjects were physically active, and their primary exercise was resistance training (Total Walking METminutes/week $1973.4 \pm 2230.4$; Total Moderate MET-minutes/week total $2587.5 \pm 2224.0$; Total Vigorous MET-minutes/week $4196.1 \pm$ 4069.1; Total Physical Activity MET-minutes / week, 8757.0 \pm 4683.2; Sitting Total Minutes/week 2074.5 \pm 925.6; Average Sitting Total Minutes/day $296.4 \pm 132.2$ ). Table A1 shows these characteristics and is presented in Appendix A.

\subsection{Trait Measures}

Trait PE was $8 \pm 1.8$ with a range of $3-10$, and trait PF was $3.6 \pm 2.6$ with a range of $0-12$. Trait ME was $7.25 \pm 2.5$ with a range of $0-10$, and trait MF was $4.2 \pm 2.6$ with a range of $0-12$. The median and interquartile ranges are shown in Table A2 presented in Appendix A.

\subsection{Diversity Measures}

Only one alpha diversity measure, Faith PD, was correlated with one trait, PF. Faith PD is the sum of the branch lengths on the phylogenetic tree connecting all members of the set. The higher the Faith PD sum, the lower the PF measure $(-0.509, p=0.022)$. These data are presented in Table A2 in Appendix A.

\subsection{Bacteria Taxa Correlated with Four Traits}

The bacteria correlated with each trait are shown in Table 1. Traits ME and PF were correlated with more than one phylum, respectively. Only trait mental energy was correlated with members of the Actinobacteria and Verrumomicrobia, while members of Proteobacteria and Bacteroidetes were only associated with trait PF. Interestingly, at least one member of the Firmicutes phyla was correlated with every trait, but only one Firmicute member, c__Clostridia;o__Clostridiales;f__Lachnospiraceae;g_Anaerostipes;s__, was correlated with more than one trait (i.e., $\mathrm{ME}, \mathrm{MF}, \mathrm{PF}$ ).

\subsection{Nutrients and Food Groups Correlated with the Four Traits}

The average fiber intake was $20.6 \pm 2.5 \mathrm{~g}$ and ranged from $3.1 \mathrm{~g}$ to $46.0 \mathrm{~g}$. Table 3 shows the significant correlations between the four traits, two nutrients (folate and lycopene), and four food groups (i.e., total dark green, red and orange, starchy, and other vegetables; dark green vegetables; grains defined as whole grains and which contain the entire grain kernel; and frankfurters, sausages, corned beef, and luncheon meat). Folate and lycopene can be found in three of the four identified food groups (i.e., total dark green, red and orange, starchy, and other vegetables; dark green vegetables; and grains defined as whole grains and which contain the entire grain kernel). Interestingly, folate and food sources rich in folate, dark green vegetables and total dark green, red and orange, starchy, and other vegetables were positively correlated with ME. Lycopene was positively correlated with both mental and physical fatigue. Processed meats like frankfurters, sausage, corned beef, and luncheon meat correlated with all four traits; negatively with ME and PE and positively with MF and PF. 
Table 1. Bacteria taxa significantly correlated with specific traits. Same colored bacteria names correlate with more than one trait.

\begin{tabular}{|c|c|c|}
\hline & $\begin{array}{l}\text { Correlation } \\
\text { Coefficient }\end{array}$ & Significance \\
\hline \multicolumn{3}{|l|}{ Trait Mental Energy } \\
\hline p_Actinobacteria & 0.469 & 0.037 \\
\hline p_Firmicutes & 0.520 & 0.019 \\
\hline p__Firmicutes;c_Bacilli;o__Turicibacterales & 0.470 & 0.037 \\
\hline p__Firmicutes;c_Bacilli;o__Turicibacterales;f_Turicibacteraceae & 0.470 & 0.037 \\
\hline p__Firmicutes;c_Bacilli;o__Turicibacterales;f__Turicibacteraceae;g__Turicibacter & 0.470 & 0.037 \\
\hline p__Firmicutes;c_Clostridia;o__Clostridiales;f__Lachnospiraceae;g__s__ & 0.461 & 0.041 \\
\hline p__Firmicutes;c_Clostridia;o_CClostridiales;f__Lachnospiraceae;g_[Ruminococcus];s_gnavus & 0.478 & 0.033 \\
\hline p__Firmicutes;c_Clostridia;o_Clostridiales;f__Lachnospiraceae;g_Anaerostipes;s__ * & 0.480 & 0.032 \\
\hline p__Firmicutes;c_CClostridia;o__Clostridiales;f_Ruminococcaceae;g_;s__ & 0.454 & 0.044 \\
\hline p__Firmicutes;c_Clostridia;o__Clostridiales;f_LLachnospiraceae;g_Coprococcus;s_catus & 0.479 & 0.032 \\
\hline p__Firmicutes;c_CClostridia;o__Clostridiales;f__Lachnospiraceae;g_Roseburia;s_faecis & 0.558 & 0.011 \\
\hline p__Verrucomicrobia;c_Verrucomicrobiae & 0.475 & 0.034 \\
\hline p__Verrucomicrobia;c_Verrucomicrobiae;o__Verrucomicrobiales & 0.475 & 0.034 \\
\hline p__Verrucomicrobia;c_Verrucomicrobiae;o__Verrucomicrobiales;f__Verrucomicrobiaceae & 0.475 & 0.034 \\
\hline $\begin{array}{l}\text { p__Verrucomicrobia;c_Verrucomicrobiae;o__Verrucomicrobiales;f__Verrucomicrobiaceae; } \\
\text { g__Akkermansia }\end{array}$ & 0.475 & 0.034 \\
\hline \multicolumn{3}{|l|}{ Trait Mental Fatigue } \\
\hline p__Firmicutes;c_Erysipelotrichi & 0.451 & 0.046 \\
\hline p__Firmicutes;c_Erysipelotrichi;o__Erysipelotrichales & 0.451 & 0.046 \\
\hline p__Firmicutes;c_Erysipelotrichi;o__Erysipelotrichales;f_Erysipelotrichaceae & 0.451 & 0.046 \\
\hline $\begin{array}{l}\text { p_Firmicutes;c_Clostridia;o__Clostridiales;f__Lachnospiraceae;g__Anaerostipes;s__ } \\
\text { Trait Physical Energy }\end{array}$ & -0.532 & 0.016 \\
\hline p__Firmicutes;c_Erysipelotrichi;o_Erysipelotrichales;f_Erysipelotrichaceae;g__Holdemania & -0.533 & 0.015 \\
\hline p__Firmicutes;c_Clostridia;o_Clostridiales;f_Lachnospiraceae;g_Dorea;s__ & -0.463 & 0.040 \\
\hline p__Firmicutes;c_Clostridia;o__Clostridiales;f__Peptostreptococcaceae;g_;s__ & -0.461 & 0.041 \\
\hline \multicolumn{3}{|l|}{ Trait Physical Fatigue } \\
\hline p_Firmicutes;c_Clostridia;o__Clostridiales;f_CChristensenellaceae;g_;s__ & -0.630 & 0.003 \\
\hline p__Firmicutes;c_CClostridia;o_Clostridiales;f_LLachnospiraceae;g_Anaerostipes;s__ & -0.448 & 0.048 \\
\hline p_Proteobacteria;c_Gammaproteobacteria;o_Pasteurellales & 0.445 & 0.049 \\
\hline p__Proteobacteria;c__Gammaproteobacteria;o_Pasteurellales;f__Pasteurellaceae & 0.445 & 0.049 \\
\hline p__Proteobacteria;c__Gammaproteobacteria;o__Pasteurellales;f_Pasteurellaceae;g_Haemophilus & 0.512 & 0.021 \\
\hline p__Bacteroidetes;c_Bacteroidia;o__Bacteroidales;f__Bacteroidaceae;g_Bacteroides;s__ & -0.451 & 0.046 \\
\hline
\end{tabular}

* Bacteria highlighted in red was found in more than one trait.

\subsection{Predicted Functional Pathways Correlated with Traits}

The functional pathways correlated with each trait are shown in Table 2. Functional metabolic pathways were predicted from the bacterial gene sequences and correlated with each trait. MF and PF did not associate with any functional pathways. Only one metabolic pathway was correlated with PE, while 19 were correlated with trait ME. Of the 19, 11 $(57 \%)$ were metabolism-correlated pathways, and 6 of these $11(55 \%)$ were involved with xenobiotics biodegradation and metabolism.

\subsection{Nutrients and Food Groups Correlated with Bacteria Correlated with the Four Traits}

Since several bacteria and food groups were correlated with at least one of the four traits, correlations were examined between the bacteria that mapped to one of the traits and the food groups correlated with at least one trait (Table 4). Interestingly, only one bacterium was correlated with each food group and most were from the Erysipelotrichi class. Only Firmicutes;c_Erysipelotrichi;o_Erysipelotrichales;f_Erysipelotrichaceae;g_Holdemania was correlated with processed meats, and, interestingly, this bacteria was negatively correlated with trait PE. 
Table 2. Predicted functional pathways significantly correlated with specific traits.

\begin{tabular}{|c|c|c|}
\hline & $\begin{array}{l}\text { Correlation } \\
\text { Coefficient }\end{array}$ & Significance \\
\hline \multicolumn{3}{|l|}{ Trait Mental Energy } \\
\hline Cellular Processes; Cell Motility; Bacterial motility proteins & 0.494 & 0.027 \\
\hline Genetic Information Processing; Replication and Repair; Non-homologous end-joining & 0.523 & 0.018 \\
\hline Human Diseases; Infectious Diseases; African trypanosomiasis & 0.501 & 0.025 \\
\hline Metabolism; Biosynthesis of Other Secondary Metabolites; Butirosin and neomycin biosynthesis & 0.445 & 0.049 \\
\hline Metabolism; Biosynthesis of Other Secondary Metabolites; Flavonoid biosynthesis & 0.531 & 0.016 \\
\hline Metabolism; Lipid Metabolism; Biosynthesis of unsaturated fatty acids & 0.470 & 0.037 \\
\hline $\begin{array}{l}\text { Metabolism; Metabolism of Terpenoids and Polyketides } \\
\text { Biosynthesis of siderophore group nonribosomal peptides }\end{array}$ & 0.450 & 0.046 \\
\hline Metabolism; Metabolism of Terpenoids and Polyketides; Carotenoid biosynthesis & 0.621 & 0.003 \\
\hline Metabolism; Xenobiotics Biodegradation and Metabolism; Benzoate degradation & 0.461 & 0.041 \\
\hline $\begin{array}{l}\text { Metabolism; Xenobiotics Biodegradation and Metabolism; Chloroalkane and } \\
\text { chloroalkene degradation }\end{array}$ & 0.470 & 0.037 \\
\hline Metabolism; Xenobiotics Biodegradation and Metabolism; Dioxin degradation & 0.464 & 0.039 \\
\hline $\begin{array}{l}\text { Metabolism; Xenobiotics Biodegradation and Metabolism; Metabolism of xenobiotics by } \\
\text { cytochrome P } 450\end{array}$ & 0.446 & 0.049 \\
\hline Metabolism; Xenobiotics Biodegradation and Metabolism; Naphthalene degradation & 0.451 & 0.046 \\
\hline Metabolism; Xenobiotics Biodegradation and Metabolism; Xylene degradation & 0.453 & 0.045 \\
\hline Organismal Systems; Digestive System; Carbohydrate digestion and absorption & 0.511 & 0.021 \\
\hline Organismal Systems; Endocrine System; Insulin signaling pathway & 0.447 & 0.048 \\
\hline Organismal Systems; Immune System; NOD-like receptor signaling pathway & 0.446 & 0.049 \\
\hline Unclassified; Cellular Processes and Signaling; Electron transfer carriers & 0.484 & 0.031 \\
\hline Unclassified; Metabolism; Lipid metabolism & 0.448 & 0.048 \\
\hline \multicolumn{3}{|l|}{ Trait Physical Energy } \\
\hline Human Diseases; Infectious Diseases; Bacterial invasion of epithelial cells & -0.604 & 0.005 \\
\hline
\end{tabular}

Table 3. Correlation of traits with nutrients and food groups from $24 \mathrm{~h}$ recall.

\begin{tabular}{|c|c|c|c|c|c|}
\hline & & $\begin{array}{l}\text { Trait Mental } \\
\text { Energy }\end{array}$ & $\begin{array}{l}\text { Trait Mental } \\
\text { Fatigue }\end{array}$ & $\begin{array}{l}\text { Trait Physical } \\
\text { Energy }\end{array}$ & $\begin{array}{c}\text { Trait Physical } \\
\text { Fatigue }\end{array}$ \\
\hline \multirow[t]{2}{*}{ Folate, food (mcg) } & Correlation & $0.465 *$ & 0.021 & 0.313 & 0.129 \\
\hline & Sig. (2-tailed) & 0.039 & 0.931 & 0.178 & 0.588 \\
\hline \multirow[t]{2}{*}{ Lycopene (mcg) } & Correlation & -0.399 & 0.505 & -0.438 & 0.503 \\
\hline & Sig. (2-tailed) & 0.081 & 0.023 & 0.053 & 0.024 \\
\hline \multirow{2}{*}{$\begin{array}{l}\text { Total dark green, red and orange, starchy, and other } \\
\text { vegetables; excludes legumes (cup eq.) }\end{array}$} & Correlation & 0.500 & -0.018 & 0.221 & 0.036 \\
\hline & Sig. (2-tailed) & 0.025 & 0.940 & 0.350 & 0.880 \\
\hline \multirow[t]{2}{*}{ Dark green vegetables (cup eq.) } & P Correlation & 0.456 & 0.052 & 0.322 & 0.187 \\
\hline & Sig. (2-tailed) & 0.043 & 0.829 & 0.166 & 0.429 \\
\hline \multirow{4}{*}{$\begin{array}{l}\text { Grains defined as whole grains and which contain the entire } \\
\text { grain kernel: bran, germ, and endosperm (oz. eq.) } \\
\text { Frankfurters, sausages, corned beef, and luncheon meat that } \\
\text { are made from beef, pork, or poultry (oz. eq.) }\end{array}$} & Correlation & -0.609 & 0.383 & -0.442 & 0.466 \\
\hline & Sig. (2-tailed) & 0.004 & 0.095 & 0.051 & 0.038 \\
\hline & Correlation & -0.790 & $0.538^{*}$ & -0.478 & 0.513 \\
\hline & Sig. (2-tailed) & $<0.0001$ & 0.014 & 0.033 & 0.021 \\
\hline
\end{tabular}

${ }^{*}$ numbers highlighted in red are significantly different.

Table 4. Correlations between significant bacteria and significant nutrient/food groups.

\begin{tabular}{|c|c|c|c|c|c|c|c|}
\hline Bacteria (All Belong to Firmicutes Phylum) & & $\begin{array}{l}\text { Folate, } \\
\text { Food } \\
\text { (mcg) }\end{array}$ & $\begin{array}{l}\text { Lycopene } \\
(\mathrm{mcg})\end{array}$ & $\begin{array}{l}\text { Total Dark } \\
\text { Green, Red and } \\
\text { Orange, Starchy, } \\
\text { and Other } \\
\text { Vegetables; } \\
\text { Excludes } \\
\text { Legumes } \\
\text { (Cup Eq.) }\end{array}$ & $\begin{array}{l}\text { Dark Green } \\
\text { Vegetables } \\
\text { (Cup Eq.) }\end{array}$ & $\begin{array}{l}\text { Grains Defined as } \\
\text { Whole Grains and } \\
\text { Which Contain the } \\
\text { Entire Grain Kernel: } \\
\text { Bran, Germ, and } \\
\text { Endosperm } \\
\text { (Oz. Eq.) }\end{array}$ & $\begin{array}{c}\text { Frankfurters, } \\
\text { Sausages, Corned } \\
\text { Beef, and Luncheon } \\
\text { Meat That Are } \\
\text { Made from Beef, } \\
\text { Pork, or Poultry } \\
\text { (Oz. Eq.) }\end{array}$ \\
\hline \multirow{2}{*}{ c_Clostridia;o_Clostridiales;__Lachnospiraceae;g_Coprococcus;s_catus } & Correlation & 0.429 & -0.354 & 0.391 & 0.491 * & 0.209 & -0.075 \\
\hline & Sig. (2-tailed) & 0.059 & 0.126 & 0.088 & 0.028 & 0.376 & 0.753 \\
\hline \multirow{2}{*}{ c_Erysipelotrichi } & Correlation & -0.281 & 0.470 & -0.277 & -0.212 & 0.344 & 0.262 \\
\hline & Sig. (2-tailed) & 0.230 & 0.037 & 0.238 & 0.370 & 0.137 & 0.264 \\
\hline \multirow{2}{*}{ c_Erysipelotrichi;o_Erysipelotrichales } & Correlation & -0.281 & 0.470 & -0.277 & -0.212 & 0.344 & 0.262 \\
\hline & Sig. (2-tailed) & 0.230 & 0.037 & 0.238 & 0.370 & 0.137 & 0.264 \\
\hline \multirow{2}{*}{ c_Erysipelotrichi;o_Erysipelotrichales;__Erysipelotrichaceae } & Correlation & $\begin{array}{l}0.281 \\
-0.281\end{array}$ & 0.470 & $\begin{array}{l}.2200 \\
-0.277\end{array}$ & -0.212 & 0.344 & 0.262 \\
\hline & Sig. (2-tailed) & 0.230 & 0.037 & 0.238 & 0.370 & 0.137 & 0.264 \\
\hline \multirow{2}{*}{ c_Erysipelotrichi;o_Erysipelotrichales;f_Erysipelotrichaceae;g_Holdemania } & Correlation & -0.268 & 0.088 & -0.339 & -0.330 & 0.455 & 0.488 \\
\hline & Sig. (2-tailed) & 0.254 & 0.713 & 0.143 & 0.155 & 0.044 & 0.029 \\
\hline
\end{tabular}

* Numbers highlighted in red are significantly different. 


\subsection{Metabolomics}

PCA analysis revealed that the subjects naturally separated into two distinct clusters (left or right, Figure 1A). Of the identified metabolites, 100 metabolites had a significantly higher relative abundance in the left group (presented in blue), including but not limited to biotin, serine, and shikimate. The right group (presented in red) had only six metabolites with a higher relative abundance, including propionyl-CoA, acetyl-CoA, and NAD+. There were no statistically significant differences in $\mathrm{PF}, \mathrm{PE}, \mathrm{MF}$, or $\mathrm{ME}$ when comparing the subjects on the left to those on the right (Figure 1B). Therefore, the separation of the clusters observed on the PCA plot cannot be attributed to traits, so distinct metabolic profiles based on $\mathrm{MF}, \mathrm{ME}, \mathrm{PF}$, and PE were not observed in the study.

A.

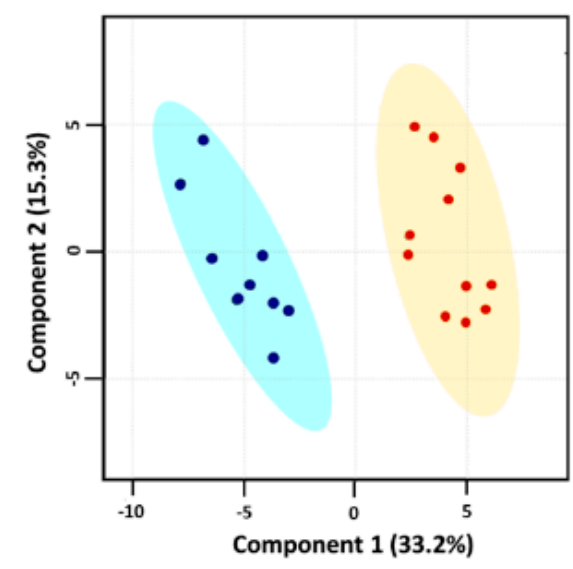

B.

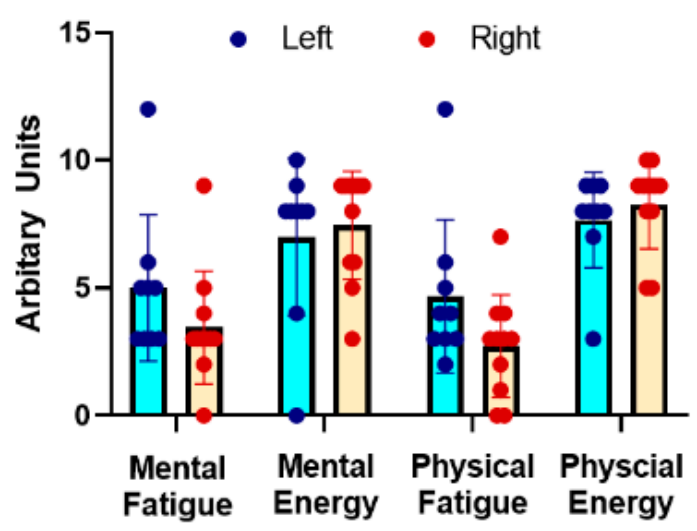

Figure 1. A principal component analysis (PCA) for the 20 survey subjects. (A) clearly shows two distinct clusters: left blue and right red. (B) shows the average \pm SD for each trait. MF, ME, PF, and PE were not significantly different between the two clusters.

\section{Discussion}

This exploratory study provides interesting evidence that gut microbiota, diet, and the corresponding metabolome may be correlated with four distinct mental and physical energy and fatigue traits. Findings provide evidence that may assist researchers when further exploring the associations between gut microbiota and trait-level mental and physical energy and fatigue. Although unique metabolic profiles were apparent in the PCA analysis, there was no evidence to attribute these differences to traits energy and fatigue. However, these findings indicate distinct bacteria may be correlated with each trait except for one, supporting previous findings that feelings of mental and physical energy and fatigue may be unique with some overlap $[13,15,16]$. In addition, only one bacterium was correlated 
with more than one trait, Anaerostipes, in the expected direction (i.e., positively correlated with mental energy and negatively correlated with mental and physical fatigue traits).

When examining bacterial functional pathways, none of the pathways were correlated with traits mental and physical fatigue; however, most of the pathways (22 out of 23) were correlated with trait mental energy. Most of the pathways fell under Metabolism (level 1) and Xenobiotic Biodegradation (level 2). Unfortunately, it is not apparent why more of these pathways were expressed in trait mental energy, which warrants further investigation. The metabolic pathway for bacterial invasion of epithelial cells was negatively correlated with trait physical energy, and again, it is not clear what this means and needs further investigation.

Only the Faith PD, a measure of alpha diversity, was negatively correlated with trait physical fatigue, suggesting that individuals who report normally feeling physically fatigued also had the lowest diversity of bacteria. The American Gut Project found that the gut microbiome is more diverse in people who eat more than 30 types of plants a week [65]. Plants are rich in fiber compared with animal-derived foods, and the Western diet, typically eaten in the USA, is high in animal protein and low in plant sources. Subjects in this study self-identified being physically active and often participating in resistance training. Their average protein intake was $136.2 \pm 15.3 \mathrm{~g}$ /day (32.2 minimum and 272 maximum). Subjects' fiber intake ranged from a low of $3 \mathrm{~g} /$ day to $46 \mathrm{~g} /$ day, and the average intake was less than the daily recommended intake (DRI) for males (38 g/day) and females (25 g/day) [66]. Only six subjects met or exceeded the DRI. For this sample, it is possible that the inadequate intake of fiber reduced the alpha diversity of their gut microbiome.

Findings from this study indicate that bacteria involved in gut homeostasis and health (Actiobacteria [67] and Firmicutes Bacilli [68]), carbohydrate metabolism (Firmicutes and Firmicutes Clostridia [69]), and glucose homeostasis (Verrucomicrobia [70]) are positively correlated with long-standing pre-disposition to ME. These findings support other biological associations with ME. For example, Dupree and colleagues [14] in a case-controlled crossover trial, reported that changes in ME were associated with decreased Annexin A1, a mediator of the glucocorticoid, cortisol. This finding aligns with the current study's findings because increased carbohydrate metabolism and improved glucose homeostasis were correlated with lower cortisol levels [60], which may influence Annexin A1 levels. Additionally, Boolani and colleagues (2019) [13] reported an association between ATP production and feelings of energy without differentiating between the physical and mental aspects of energy. Curiously, in the current study, trait PE was negatively correlated with Dorea and Holdemania, both of which have been correlated with high fiber diets [71], and lower levels of these have been correlated with higher blood pressure [72].

Trait MF was primarily correlated with Firmicutes Erysipelotrichi, bacteria primarily associated with TNF- $\alpha$ [73], a proinflammatory cytokine. These findings support the work by Loy and colleagues (2018) [12], who reported that proinflammatory cytokines are associated with feelings of fatigue only, without differentiating between the mental and physical aspects of fatigue. Findings from the current study further support the idea of inflammation being correlated with feelings of fatigue in that trait PF was correlated with Proteobacteria Gammaproteobacteria, which are reported to be higher in individuals who have inflammatory bowel disease [74-76]. Taken together, this study's findings support the work by Loy and colleagues (2018) [12] in that fatigue was associated with inflammation.

Interestingly, Anaerostipes was correlated with trait $\mathrm{ME}, \mathrm{MF}$, and $\mathrm{PF}$ in the anticipated direction. Higher levels of Anaerostipes are associated with activation of fatty acid oxidation, synthesis, and lipolysis inhibition, which in turn decreases circulating lipid plasma levels and body weight [76]. It also suppresses colon inflammation and can downregulate insulin signal transduction in adipose tissue. Although this study did not differentiate which function the bacteria serve in each trait, based on previous findings [12] and the other bacteria associated with the traits, the authors hypothesize that Anaerostipes' anti-inflammatory function may be associated with MF and PF. In contrast, the metabolic function of the Anaerostipes bacteria may be correlated with trait ME. 
When examining functional pathways, a majority were positively correlated with trait $\mathrm{ME}$, while the functional pathway correlated with bacterial invasion of epithelial cells was negatively correlated with trait PE. Many of the functional pathways correlated with trait ME were metabolism-related pathways, suggesting that those who normally feel mentally energetic may produce gut substrates that impact metabolism in the gut and possibly the host. These findings may be important for nutrition science researchers because those reporting high trait ME may be hyper- or hypo-responders to some nutritional interventions (i.e., cocoa). Trait moods have been noted to influence acute responses to caffeine [23]; however, none of the pathways that were significant in the current study were correlated with methylxanthine metabolism.

The processed meats (frankfurters, sausages, corned beef, and luncheon meat that are made from beef, pork, or poultry) food group was the only one correlated with all four traits. These foods are also considered ultra-processed foods. Hall and colleagues [77] demonstrated in a randomized controlled trial that diets rich in ultra-processed foods promote excess calorie intake and weight gain. Limiting these foods may be an effective strategy to prevent obesity. Obese people often experience fatigue and decreased physical endurance [78]. The other identified foods groups in the current study were plant-based. As previously discussed, plants contain many substances that can be metabolized by the gut microbiota and impact host physiology. It is possible that the observed changes in the metabolome can be attributed to diet rather than trait because diet is known to alter the gut microbiome, which then leads to changes in the available small molecules, or nutrients, produced by the gut microbiota. Future studies should consider and account for the types of foods subjects eat.

\section{Limitations}

As with all studies, this study is not without limitations. The primary limitation was the small sample size and the lack of significant findings when adjusting for multiple comparisons. However, since this was an exploratory study, and because this study was intended to be illustrative rather than definitive, the authors were unable to complete power and sample size calculations a priori. Nevertheless, these preliminary data show interesting findings, which can serve as foundational evidence for future investigations looking to determine the role of the gut microbiota in feelings of mental and physical energy and fatigue. Additionally, these study findings were limited to a single sample of healthy young adults and cannot be extrapolated to individuals who may have a diagnosis of diseases such as Inflammatory Bowel Syndrome (IBS), which has been known to influence gut microbiota composition. Future studies are recommended to add comparator subgroups with differing diagnoses so the relationships in this study can be compared by health status. Another potential limitation to this study is that stool samples and trait measures were collected two years apart. Future studies should consider collecting all microbiome and selfreported data from participants in closer temporal proximity. However, there is significant evidence that suggests that trait mental and physical energy and fatigue maintain temporal stability for as long as one year $[15,16,47,79]$. Additionally, there is scientific evidence that gut microbiota maintains temporal stability throughout adulthood [80,81]. However, exceptions are associated with inflammatory bowel syndrome [82,83], obesity [84,85], irritable bowel syndrome [86,87] (Jeffery et al., 2012; DuPont 2014), and type 2 diabetes [88] (Larsen et al., 2010). Therefore, participants who had any of these diseases/syndromes were excluded from the current study. Antibiotic therapy can also disrupt the gut microbiome, so individuals who had taken antibiotics less than three months prior to the study were also excluded. Fu et al. [89] examined the reliability and biological stability of fecal samples collected every six months for two years. They found that a single sample is sufficient to capture the majority of the variation in fecal microbiome from $16 \mathrm{~S}$ rRNA gene sequencing. Multiple samples are needed for rare or less-abundant taxa, which are not reported in the current study. Finally, while 170 metabolites were identified in the fecal samples in this study; details were only provided for those that significantly differed in bivariate 
analyses and were included in subsequent analyses. While short-chain fatty acids were not measured for this study, future studies should examine these metabolites as related to trait mental and physical energy and fatigue.

\section{Conclusions}

The objective of this exploratory study was to identify potential correlations between gut microbiota and trait (long-standing pre-disposition to) mental and physical energy and fatigue, which can be used to guide future research. These findings provide evidence that the four traits (i.e., mental energy (ME), mental fatigue (MF), physical energy (PE), and physical fatigue (PF)) may have unique yet overlapping gut bacteria profiles. For example, the bacteria most often correlated with feelings of energy perform metabolic functions, while bacteria most often correlated with feelings of fatigue are associated with inflammation. This study suggests the need to explore the role of gut microbiota in understanding long-standing feelings of energy and fatigue among healthy young individuals.

Author Contributions: Conceptualization, L.O.B., K.M.G., K.S.O. and A.B.; methodology, L.O.B., A.B., C.J.C., H.F.C. and S.R.C.; formal analysis, L.O.B., A.B., C.M.T., M.L., S.E.D., C.J.C., H.F.C., M.L.S. and S.R.C.; resources, L.O.B. and A.B.; data curation, L.O.B., C.J.C. and A.B.; writing-original draft preparation, L.O.B., A.B. and C.J.C.; writing-review and editing, L.O.B., K.S.O., K.M.G., C.M.T., M.L., S.E.D., C.J.C., H.F.C., S.R.C., M.L.S. and A.B.; funding acquisition, L.O.B., K.M.G. and K.S.O. All authors have read and agreed to the published version of the manuscript.

Funding: This research was funded by American Public University System.

Institutional Review Board Statement: The study was conducted according to the guidelines of the Declaration of Helsinki and approved by the Institutional Review Board (or Ethics Committee) of the American Public University System (protocol code 2021-045-OL, 14 April 2021).

Informed Consent Statement: Informed consent was obtained from all subjects involved in the study.

Data Availability Statement: The data presented in this study are available on request from the corresponding author.

Conflicts of Interest: The authors declare no conflict of interest.

\section{Appendix A}

Table A1. Subject information.

\begin{tabular}{ccc}
\hline Variable & Median & Interquartile Range \\
\hline Age (years) & 31 & 7 \\
Sex (Males, Females) & 14,6 & \\
Weight (lbs) & 180 & 54 \\
Height (inches) & 68 & 7 \\
BMI (kg/m ${ }^{2}$ ) & 25.3 & 5.1 \\
Total Walking MET-min/week & 1402.5 & 2883.4 \\
Total Moderate MET-min/week total & 1560.0 & 3532.5 \\
Total Vigorous MET-min/week & 2880.0 & 2520.0 \\
Total Physical Activity MET-min/week & 7939.5 & 5711.6 \\
Sitting Total Minutes/week & 2220.0 & 1245.0 \\
Average Sitting Total Minutes/day & 317.1 & 177.9 \\
Trait Physical Energy & 8.0 & 3.0 \\
Trait Physical Fatigue & 3.0 & 2.0 \\
Trait Mental Energy & 8.5 & 1 \\
Trait Mental Fatigue & 3.0 & 1.8 \\
\hline
\end{tabular}


Table A2. Correlation of trait measures with four alpha diversity measures.

\begin{tabular}{llcccc}
\hline $\begin{array}{c}\text { Alpha } \\
\text { Diversity } \\
\text { Measure }\end{array}$ & & $\begin{array}{c}\text { Trait Mental } \\
\text { Energy }\end{array}$ & $\begin{array}{c}\text { Trait Mental } \\
\text { Fatigue }\end{array}$ & $\begin{array}{c}\text { Trait } \\
\text { Physical } \\
\text { Energy }\end{array}$ & $\begin{array}{c}\text { Trait } \\
\text { Physical } \\
\text { Fatigue }\end{array}$ \\
\hline \multirow{2}{*}{ Evenness } & $\begin{array}{c}\text { Correlation } \\
\text { Coefficient }\end{array}$ & 0.099 & -0.401 & 0.092 & -0.387 \\
& $\begin{array}{c}\text { Significance } \\
\text { Shannon }\end{array}$ & 0.679 & 0.079 & 0.700 & 0.092 \\
& $\begin{array}{l}\text { Correlation } \\
\text { Coefficient }\end{array}$ & 0.293 & -0.330 & 0.199 & -0.432 \\
& $\begin{array}{l}\text { Significance } \\
\text { Observed Otus }\end{array}$ & 0.211 & 0.156 & 0.401 & 0.057 \\
Faith PD & $\begin{array}{c}\text { Correlation } \\
\text { Coefficient }\end{array}$ & 0.432 & -0.185 & 0.331 & -0.390 \\
& $\begin{array}{l}\text { Significance } \\
\text { Correlation }\end{array}$ & 0.057 & 0.435 & 0.154 & 0.089 \\
& $\begin{array}{c}\text { Coefficient } \\
\text { Significance }\end{array}$ & 0.293 & -0.367 & 0.247 & -0.509 \\
\hline
\end{tabular}

\section{References}

1. Chen, M.K. The epidemiology of self-perceived fatigue among adults. Prev. Med. 1986, 15, 74-81. [CrossRef]

2. Cunningham, T.J.; Ford, E.S.; Chapman, D.P.; Liu, Y.; Croft, J.B. Independent and joint associations of race/ethnicity and educational attainment with sleep-related symptoms in a population-based US sample. Prev. Med. 2015, 77, 99-105. [CrossRef]

3. Lewis, G.; Wessely, S. The epidemiology of fatigue: More questions than answers. J. Epidemiol. Community Health 1992, $46,92-97$. [CrossRef]

4. Ricci, J.A.; Chee, E.; Lorandeau, A.L.; Berger, J. Fatigue in the U.S. workforce: Prevalence and implications for lost productive work time. J. Occup. Environ. Med. 2007, 49, 1-10. [CrossRef]

5. Taylor, A.H.; Dorn, L. Stress, fatigue, health, and risk of road traffic accidents among professional drivers: The contribution of physical inactivity. Annu. Rev. Public Health 2006, 27, 371-391. [CrossRef]

6. Van Drongelen, A.; Boot, C.R.; Hlobil, H.; Smid, T.; van der Beek, A.J. Risk factors for fatigue among airline pilots. Int. Arch. Occup. Environ. Health 2017, 90, 39-47. [CrossRef]

7. Samkoff, J.S.; Jacques, C.H. A review of studies concerning effects of sleep deprivation and fatigue on residents' performance. Acad. Med. 1991, 66, 687-693. [CrossRef]

8. Bakker, R.J.; van de Putte, E.M.; Kuis, W.; Sinnema, G. Risk factors for persistent fatigue with significant school absence in children and adolescents. Pediatrics 2009, 124, e89-e95. [CrossRef]

9. Fukuda, S.; Yamano, E.; Joudoi, T.; Mizuno, K.; Tanaka, M.; Kawatani, J.; Takano, M.; Tomoda, A.; Imai-Matsumura, K.; Miike, T.; et al. Effort-reward imbalance for learning is associated with fatigue in school children. Behav. Med. 2010, 36, 53-62. [CrossRef]

10. Verbrugge, L.M.; Ascione, F.J. Exploring the iceberg. Common symptoms and how people care for them. Med. Care 1987, 25, 539-569. [CrossRef]

11. Hjollund, N.H.; Andersen, J.H.; Bech, P. Assessment of fatigue in chronic disease: A bibliographic study of fatigue measurement scales. Health Qual. Life Outcomes 2007, 5, 12. [CrossRef]

12. Loy, B.D.; Cameron, M.H.; O'Connor, P.J. Perceived fatigue and energy are independent unipolar states: Supporting evidence. Med. Hypotheses 2018, 113, 46-51. [CrossRef]

13. Boolani, A.; O'Connor, P.J.; Reid, J.; Ma, S.; Mondal, S. Predictors of feelings of energy differ from predictors of fatigue. Fatigue Biomed. Health Behav. 2019, 7, 12-28. [CrossRef]

14. Dupree, E.J.; Goodwin, A.; Darie, C.C.; Boolani, A. A Pilot Exploratory Proteomics Investigation of Mental Fatigue and Mental Energy. Adv. Exp. Med. Biol. 2019, 1140, 601-611.

15. Manierre, M.; Jansen, E.; Boolani, A. Sleep quality and sex modify the relationships between trait energy and fatigue on state energy and fatigue. PLoS ONE 2020, 15, e0227511. [CrossRef]

16. Boolani, A.; Manierre, M. An exploratory multivariate study examining correlates of trait mental and physical fatigue and energy. Fatigue Biomed. Health Behav. 2019, 7, 29-40. [CrossRef]

17. Boolani, A.; Sur, S.; Yang, D.; Avolio, A.; Goodwin, A.; Mondal, S.; Fulk, G.; Towler, C.; Lee Smith, M. Six Minutes of Physical Activity Improves Mood in Older Adults: A Pilot Study. J. Geriatr. Phys. Ther. 2021, 44, 18-24. [CrossRef]

18. O'Connor, P.J.; Puetz, T.W. Chronic physical activity and feelings of energy and fatigue. Med. Sci. Sports Exerc. 2005, 37, 299-305. [CrossRef]

19. Loy, B.D.; O'Connor, P.J.; Dishman, R.K. The effect of a single bout of exercise on energy and fatigue states: A systematic review and meta-analysis. Fatigue Biomed. Health Behav. 2013, 1, 223-242. [CrossRef]

20. Miller, M.; Lee-Chambers, J.; Cooper, B.; Boolani, A.; Jansen, E. Associations between physical activity and energy and fatigue depend on sleep quality. Fatigue Biomed. Health Behav. 2020, 8, 193-204. [CrossRef] 
21. Maridakis, V.; Herring, M.P.; O'Connor, P.J. Sensitivity to change in cognitive performance and mood measures of energy and fatigue in response to differing doses of caffeine or breakfast. Int. J. Neurosci. 2009, 119, 975-994. [CrossRef]

22. Boolani, A.; Lindheimer, J.B.; Loy, B.D.; Crozier, S.; O'Connor, P.J. Acute effects of brewed cocoa consumption on attention, motivation to perform cognitive work and feelings of anxiety, energy and fatigue: A randomized, placebo-controlled crossover experiment. BMC Nutr. 2017, 3, 8. [CrossRef]

23. Fuller, D.T.; Smith, M.L.; Boolani, A. Trait Energy and Fatigue Modify the Effects of Caffeine on Mood, Cognitive and Fine-Motor Task Performance: A Post-Hoc Study. Nutrients 2021, 13, 412. [CrossRef]

24. Eshragh, J.; Dhruva, A.; Paul, S.M.; Cooper, B.A.; Mastick, J.; Hamolsky, D.; Levine, J.D.; Miaskowski, C.; Kober, K.M. Associations between Neurotransmitter Genes and Fatigue and Energy Levels in Women after Breast Cancer Surgery. J. Pain Symptom Manag. 2017, 53, 67-84.e7. [CrossRef]

25. Eckburg, P.B.; Bik, E.M.; Bernstein, C.N.; Purdom, E.; Dethlefsen, L.; Sargent, M.; Gill, S.R.; Nelson, K.E.; Relman, D.A. Diversity of the human intestinal microbial flora. Science 2005, 308, 1635-1638. [CrossRef]

26. Qin, J.; Li, R.; Raes, J.; Arumugam, M.; Burgdorf, K.S.; Manichanh, C.; Nielsen, T.; Pons, N.; Levenez, F.; Yamada, T.; et al. A human gut microbial gene catalogue established by metagenomic sequencing. Nature 2010, 464, 59-65. [CrossRef]

27. Conlon, M.A.; Bird, A.R. The impact of diet and lifestyle on gut microbiota and human health. Nutrients 2014, 7, 17-44. [CrossRef]

28. Backhed, F.; Ley, R.E.; Sonnenburg, J.L.; Peterson, D.A.; Gordon, J.I. Host-bacterial mutualism in the human intestine. Science 2005, 307, 1915-1920. [CrossRef]

29. Grenham, S.; Clarke, G.; Cryan, J.F.; Dinan, T.G. Brain-gut-microbe communication in health and disease. Front. Physiol. 2011, 2, 94. [CrossRef]

30. Das, B.; Nair, G.B. Homeostasis and dysbiosis of the gut microbiome in health and disease. J. Biosci. 2019, 44, 1-8. [CrossRef]

31. Cryan, J.F.; O'Mahony, S.M. The microbiome-gut-brain axis: From bowel to behavior. Neurogastroenterol. Motil. 2011, $23,187-192$. [CrossRef]

32. Makki, K.; Deehan, E.C.; Walter, J.; Bäckhed, F. The Impact of Dietary Fiber on Gut Microbiota in Host Health and Disease. Cell Host Microbe 2018, 23, 705-715. [CrossRef]

33. Tremaroli, V.; Bäckhed, F. Functional interactions between the gut microbiota and host metabolism. Nature 2012, 489, 242-249. [CrossRef]

34. Wu, G.D.; Chen, J.; Hoffmann, C.; Bittinger, K.; Chen, Y.Y.; Keilbaugh, S.A.; Bewtra, M.; Knights, D.; Walters, W.A.; Knight, R.; et al. Linking long-term dietary patterns with gut microbial enterotypes. Science 2011, 334, 105-108. [CrossRef]

35. Koutsos, A.; Tuohy, K.M.; Lovegrove, J.A. Apples and cardiovascular health-is the gut microbiota a core consideration? Nutrients 2015, 7, 3959-3998. [CrossRef]

36. O'Connor, P.J.; Kennedy, D.O.; Stahl, S. Mental energy: Plausible neurological mechanisms and emerging research on the effects of natural dietary compounds. Nutr. Neurosci. 2019, 24, 850-864. [CrossRef]

37. Baguley, B.J.; Skinner, T.L.; Jenkins, D.G.; Wright, O.R.L. Mediterranean-style dietary pattern improves cancer-related fatigue and quality of life in men with prostate cancer treated with androgen deprivation therapy: A pilot randomised control trial. Clin. Nutr. 2021, 40, 245-254. [CrossRef]

38. Hajjar, J.; Mendoza, T.; Zhang, L.; Fu, S.; Piha-Paul, S.A.; Hong, D.S.; Janku, F.; Karp, D.D.; Ballhausen, A.; Gong, J.; et al Associations between the gut microbiome and fatigue in cancer patients. Sci. Rep. 2021, 11, 5847. [CrossRef]

39. Giloteaux, L.; Goodrich, J.K.; Walters, W.A.; Levine, S.M.; Ley, R.E.; Hanson, M.R. Reduced diversity and altered composition of the gut microbiome in individuals with myalgic encephalomyelitis/chronic fatigue syndrome. Microbiome 2016, 4, 30. [CrossRef]

40. Xiao, C.; Fedirko, V.; Beitler, J.; Bai, J.; Peng, G.; Zhou, C.; Gu, J.; Zhao, H.; Lin, I.H.; Chico, C.E.; et al. The role of the gut microbiome in cancer-related fatigue: Pilot study on epigenetic mechanisms. Support Care Cancer 2021, 29, 3173-3182. [CrossRef]

41. Mandarano, A.H.; Giloteaux, L.; Keller, B.A.; Levine, S.M.; Hanson, M.R. Eukaryotes in the gut microbiota in myalgic encephalomyelitis/chronic fatigue syndrome. PeerJ 2018, 6, e4282. [CrossRef]

42. Nuzzo, R. Scientific method: Statistical errors. Nature 2014, 506, 150-152. [CrossRef]

43. Loy, B.D.; O'Connor, P.J. The effect of histamine on changes in mental energy and fatigue after a single bout of exercise. Physiol. Behav. 2016, 153, 7-18. [CrossRef]

44. Bigelman, K.A.; Chapman, D.P.; Freese, E.C.; Trilk, J.L.; Cureton, K.J. Effects of 6 weeks of quercetin supplementation on energy, fatigue, and sleep in ROTC cadets. Mil. Med. 2011, 176, 565-572. [CrossRef]

45. Bossie, H.M.; Willingham, T.B.; Schoick, R.A.V.; O'Connor, P.J.; McCully, K.K. Mitochondrial capacity, muscle endurance, and low energy in friedreich ataxia. Muscle Nerve 2017, 56, 773-779. [CrossRef]

46. Boolani, A.; Fuller, D.T.; Mondal, S.; Wilkinson, T.; Darie, C.C.; Gumpricht, E. Caffeine-Containing, Adaptogenic-Rich Drink Modulates the Effects of Caffeine on Mental Performance and Cognitive Parameters: A Double-Blinded, Placebo-Controlled, Randomized Trial. Nutrients 2020, 12, 1922. [CrossRef]

47. Boolani, A.A.A.; Barrios, N.; Sames, C. Association between trait energy and fatigue and aquatic functional tests: An exploratory study. J. Aquat. Phys. Ther. 2021, in press.

48. Subar, A.F.; Kirkpatrick, S.I.; Mittl, B.; Zimmerman, T.P.; Thompson, F.E.; Bingley, C.; Willis, G.; Islam, N.G.; Baranowski, T.; McNutt, S.; et al. The Automated Self-Administered 24-hour dietary recall (ASA24): A resource for researchers, clinicians, and educators from the National Cancer Institute. J. Acad. Nutr. Diet. 2012, 112, 1134-1137. [CrossRef] 
49. Callahan, B.J.; McMurdie, P.J.; Rosen, M.J.; Han, A.W.; Johnson, A.J.; Holmes, S.P. DADA2: High-resolution sample inference from Illumina amplicon data. Nat. Methods 2016, 13, 581-583. [CrossRef]

50. Katoh, K.; Standley, D.M. MAFFT multiple sequence alignment software version 7: Improvements in performance and usability Mol. Biol. Evol. 2013, 30, 772-780. [CrossRef]

51. Price, M.N.; Dehal, P.S.; Arkin, A.P. FastTree 2-approximately maximum-likelihood trees for large alignments. PLoS ONE 2010, 5, e9490. [CrossRef]

52. McDonald, D.; Price, M.N.; Goodrich, J.; Nawrocki, E.P.; DeSantis, T.Z.; Probst, A.; Andersen, G.L.; Knight, R.; Hugenholtz, P. An improved Greengenes taxonomy with explicit ranks for ecological and evolutionary analyses of bacteria and archaea. ISME J. 2012, 6, 610-618. [CrossRef]

53. Bolyen, E.; Rideout, J.R.; Dillon, M.R.; Bokulich, N.A.; Abnet, C.C.; Al-Ghalith, G.A.; Alexander, H.; Alm, E.J.; Arumugam, M.; Asnicar, F.; et al. Reproducible, interactive, scalable and extensible microbiome data science using QIIME 2. Nat. Biotechnol. 2019, 37, 852-857. [CrossRef]

54. $\quad$ Langille, M.G.; Zaneveld, J.; Caporaso, J.G.; McDonald, D.; Knights, D.; Reyes, J.A.; Clemente, J.C.; Burkepile, D.E.; Vega Thurber, R.L.; Knight, R.; et al. Predictive functional profiling of microbial communities using 16S rRNA marker gene sequences. Nat. Biotechnol. 2013, 31, 814-821. [CrossRef]

55. Parks, D.H.; Beiko, R.G. Identifying biologically relevant differences between metagenomic communities. Bioinformatics 2010, 26, 715-721. [CrossRef]

56. Joshua, D.; Rabinowitz, E.K. Acidic acetonitrile for cellular metabolome extraction from Escherichia coli. Anal. Chem. 2007, 79, 6167-6173.

57. Lu, W.; Clasquin, M.F.; Melamud, E.; Amador-Noguez, D.; Caudy, A.A.; Rabinowitz, J.D. Metabolomic Analysis via ReversedPhase Ion-Pairing Liquid Chromatography Coupled to a Stand Alone Orbitrap Mass Spectrometer. Anal. Chem. 2010, 82, 3212-3221. [CrossRef]

58. Stough, J.M.A.; Dearth, S.P.; Denny, J.E.; LeCleir, G.R.; Schmidt, N.W.; Campagna, S.R.; Wilhelm, S.W. Functional Characteristics of the Gut Microbiome in C57BL/6 Mice Differentially Susceptible to Plasmodium yoelii. Front. Microbiol. 2016, 7, 1520. [CrossRef]

59. Martens, L.; Chambers, M.; Sturm, M.; Kessner, D.; Levander, F.; Shofstahl, J.; Tang, W.H.; Römpp, A.; Neumann, S.; Pizarro, A.D.; et al. mzML-a community standard for mass spectrometry data. Mol. Cell. Proteom. MCP 2011, 10, R110.000133. [CrossRef]

60. Clasquin, M.F.; Melamud, E.; Rabinowitz, J.D. LC-MS data processing with MAVEN: A metabolomic analysis and visualization engine. Curr. Protoc. Bioinform. 2012, 37, 14.11.1-14.11.23.

61. Melamud, E.; Vastag, L.; Rabinowitz, J.D. Metabolomic Analysis and Visualization Engine for LC-MS Data. Anal. Chem. 2010, 82, 9818-9826. [CrossRef] [PubMed]

62. De Livera, A.M.; Olshansky, G.; Simpson, J.A.; Creek, D.J. NormalizeMets: Assessing, selecting and implementing statistical methods for normalizing metabolomics data. Metabolomics 2018, 14, 54. [CrossRef] [PubMed]

63. Misra, B.B. Data normalization strategies in metabolomics: Current challenges, approaches, and tools. Eur. J. Mass Spectrom. 2020, 26, 165-174. [CrossRef] [PubMed]

64. Pang, Z.; Chong, J.; Zhou, G.; de Lima Morais, D.A.; Chang, L.; Barrette, M.; Gauthier, C.; Jacques, P.-É.; Li, S.; Xia, J. MetaboAnalyst 5.0: Narrowing the gap between raw spectra and functional insights. Nucleic Acids Res. 2021, 49, W388-W396. [CrossRef] [PubMed]

65. McDonald, D.; Hyde, E.; Debelius, J.W.; Morton, J.T.; Gonzalez, A.; Ackermann, G.; Aksenov, A.A.; Behsaz, B.; Brennan, C.; Chen, Y.; et al. American Gut: An Open Platform for Citizen Science Microbiome Research. mSystems 2018, 3, e00031-18. [CrossRef]

66. Meyers, L.D.; Hellwig, J.P.; Otten, J.J. (Eds.) Dietary Reference Intakes: The Essential Guide to Nutrient Requirements; The National Academies Press: Washington, DC, USA, 2006; p. 1344.

67. Binda, C.; Lopetuso, L.R.; Rizzatti, G.; Gibiino, G.; Cennamo, V.; Gasbarrini, A. Actinobacteria: A relevant minority for the maintenance of gut homeostasis. Dig. Liver Dis. 2018, 50, 421-428. [CrossRef]

68. Ilinskaya, O.N.; Ulyanova, V.V.; Yarullina, D.R.; Gataullin, I.G. Secretome of Intestinal Bacilli: A Natural Guard against Pathologies. Front. Microbiol. 2017, 8, 1666. [CrossRef]

69. Bergstrom, A.; Skov, T.H.; Bahl, M.I.; Roager, H.M.; Christensen, L.B.; Ejlerskov, K.T.; Molgaard, C.; Michaelsen, K.F.; Licht, T.R. Establishment of intestinal microbiota during early life: A longitudinal, explorative study of a large cohort of Danish infants. Appl. Environ. Microbiol. 2014, 80, 2889-2900. [CrossRef]

70. Faintuch, J.; Faintuch, S. Microbiome and Metabolome in Diagnosis, Therapy, and Other Strategic Applications; Elsevier Inc.: Amsterdam, The Netherlands, 2019; p. 472.

71. Gomez-Arango, L.F.; Barrett, H.L.; Wilkinson, S.A.; Callaway, L.K.; McIntyre, H.D.; Morrison, M.; Dekker Nitert, M. Low dietary fiber intake increases Collinsella abundance in the gut microbiota of overweight and obese pregnant women. Gut Microbes 2018, 9, 189-201. [CrossRef]

72. Gomez-Arango, L.F.; Barrett, H.L.; McIntyre, H.D.; Callaway, L.K.; Morrison, M.; Dekker Nitert, M.; Group, S.T. Increased Systolic and Diastolic Blood Pressure Is Associated with Altered Gut Microbiota Composition and Butyrate Production in Early Pregnancy. Hypertension 2016, 68, 974-981. [CrossRef]

73. Dinh, D.M.; Volpe, G.E.; Duffalo, C.; Bhalchandra, S.; Tai, A.K.; Kane, A.V.; Wanke, C.A.; Ward, H.D. Intestinal microbiota, microbial translocation, and systemic inflammation in chronic HIV infection. J. Infect. Dis. 2015, 211, 19-27. [CrossRef] [PubMed] 
74. Frank, D.N.; St Amand, A.L.; Feldman, R.A.; Boedeker, E.C.; Harpaz, N.; Pace, N.R. Molecular-phylogenetic characterization of microbial community imbalances in human inflammatory bowel diseases. Proc. Natl. Acad. Sci. USA 2007, 104, 13780-13785. [CrossRef]

75. Langgartner, D.; Peterlik, D.; Foertsch, S.; Fuchsl, A.M.; Brokmann, P.; Flor, P.J.; Shen, Z.; Fox, J.G.; Uschold-Schmidt, N.; Lowry, C.A.; et al. Individual differences in stress vulnerability: The role of gut pathobionts in stress-induced colitis. Brain Behav. Immun. 2017, 64, 23-32. [CrossRef] [PubMed]

76. Vacca, M.; Celano, G.; Calabrese, F.M.; Portincasa, P.; Gobbetti, M.; De Angelis, M. The Controversial Role of Human Gut Lachnospiraceae. Microorganisms 2020, 8, 573. [CrossRef]

77. Hall, K.D.; Ayuketah, A.; Brychta, R.; Cai, H.; Cassimatis, T.; Chen, K.Y.; Chung, S.T.; Costa, E.; Courville, A.; Darcey, V.; et al. Ultra-Processed Diets Cause Excess Calorie Intake and Weight Gain: An Inpatient Randomized Controlled Trial of Ad Libitum Food Intake. Cell Metab. 2019, 30, 67-77.e3. [CrossRef] [PubMed]

78. Wlodek, D.; Gonzales, M. Decreased energy levels can cause and sustain obesity. J. Theor. Biol. 2003, 225, 33-44. [CrossRef]

79. Pastier, N.; Jansen, E.; Boolani, A. Sleep quality in relation to trait energy and fatigue: An exploratory study of healthy young adults. Sleep Sci. 2022, e-pub ahead of print.

80. Jeffery, I.B.; Lynch, D.B.; O’Toole, P.W. Composition and temporal stability of the gut microbiota in older persons. ISME J. 2016 10, 170-182. [CrossRef] [PubMed]

81. Fassarella, M.; Blaak, E.E.; Penders, J.; Nauta, A.; Smidt, H.; Zoetendal, E.G. Gut microbiome stability and resilience: Elucidating the response to perturbations in order to modulate gut health. Gut 2021, 70, 595-605. [CrossRef]

82. Rigottier-Gois, L. Dysbiosis in inflammatory bowel diseases: The oxygen hypothesis. ISME J. 2013, 7, 1256-1261. [CrossRef]

83. Gevers, D.; Kugathasan, S.; Denson, L.A.; Vazquez-Baeza, Y.; Van Treuren, W.; Ren, B.; Schwager, E.; Knights, D.; Song, S.J.; Yassour, M.; et al. The treatment-naive microbiome in new-onset Crohn's disease. Cell Host Microbe 2014, 15, 382-392. [CrossRef] [PubMed]

84. Ley, R.E.; Turnbaugh, P.J.; Klein, S.; Gordon, J.I. Microbial ecology: Human gut microbes associated with obesity. Nature 2006, 444, 1022-1023. [CrossRef]

85. Parekh, P.J.; Arusi, E.; Vinik, A.I.; Johnson, D.A. The role and influence of gut microbiota in pathogenesis and management of obesity and metabolic syndrome. Front. Endocrinol. 2014, 5, 47. [CrossRef] [PubMed]

86. Jeffery, I.B.; O'Toole, P.W.; Ohman, L.; Claesson, M.J.; Deane, J.; Quigley, E.M.; Simren, M. An irritable bowel syndrome subtype defined by species-specific alterations in faecal microbiota. Gut 2012, 61, 997-1006. [CrossRef]

87. Dupont, H.L. Review article: Evidence for the role of gut microbiota in irritable bowel syndrome and its potential influence on therapeutic targets. Aliment. Pharmacol. Ther. 2014, 39, 1033-1042. [CrossRef] [PubMed]

88. Larsen, N.; Vogensen, F.K.; van den Berg, F.W.; Nielsen, D.S.; Andreasen, A.S.; Pedersen, B.K.; Al-Soud, W.A.; Sorensen, S.J.; Hansen, L.H.; Jakobsen, M. Gut microbiota in human adults with type 2 diabetes differs from non-diabetic adults. PLoS ONE 2010, 5, e9085. [CrossRef] [PubMed]

89. Fu, B.C.; Randolph, T.W.; Lim, U.; Monroe, K.R.; Cheng, I.; Wilkens, L.R.; Le Marchand, L.; Lampe, J.W.; Hullar, M.A.J. Temporal Variability and Stability of the Fecal Microbiome: The Multiethnic Cohort Study. Cancer Epidemiol. Biomark. Prev. 2019, 28, 154-162. [CrossRef] 